\title{
LOGARITHMIC UNIFORM DISTRIBUTION OF $(\alpha n+\beta \log n)$
}

R. F. TICHY and G. TURNWALD

\section{Introduction}

A sequence $\omega=\left(x_{n}\right)_{n=1}^{\infty}$ of real numbers is said to be uniformly distributed modulo 1 if the proportion of indices $n \leq N$ such that the fractional parts $\left\{x_{n}\right\}$ are contained in an interval $I \subseteq[0,1)$ is asymptotically equal to the length of $I$. Put $\chi(x ; y)=1$ for $\{y\}<x$ and $\chi(x ; y)=0$ otherwise; then $\omega$ is uniformly distributed if and only if

$$
\lim _{N \rightarrow \infty} \frac{1}{N} \sum_{n=1}^{N} \chi\left(x ; x_{n}\right)=x \text { for } 0<x<1 .
$$

It is well known (cf. the monographs [1] and [3] ) that (1) is equivalent to

$$
\lim _{N \rightarrow \infty} D_{N}^{*}(\omega)=0
$$

where

$$
D_{N}^{*}(\omega)=\sup _{0 \leqslant x<1}\left|\frac{1}{N} \sum_{n=1}^{N} \chi\left(x ; x_{n}\right)-x\right|
$$

denotes the discrepancy of the sequence $\omega$. The systematic study of uniformly distributed sequences was initiated by $\mathrm{H}$. Weyl[ [9]. Well known examples of uniformly distributed sequences are $(\alpha n)$ with irrational $\alpha$ and $(\sqrt{n})$; $(\log n)$ is known not to be uniformly distributed, but Tsuji [8] proved that

$$
\lim _{N \rightarrow \infty} \frac{1}{\sum_{n=1}^{N} \frac{1}{n}} \sum_{n=1}^{N} \frac{1}{n} \chi\left(x ; x_{n}\right)=x \quad(0<x<1)
$$

for $x_{n}=\log n$. A sequence $\omega=\left(x_{n}\right)$ with this property is said to be uniformly distributed with respect to the logarithmic mean. This is equivalent to

$$
\lim _{N \rightarrow \infty} D_{N}(\omega)=0
$$

Received October 1, 1985. Revised May 13, 1986. 
where

$$
D_{N}(\omega)=\sup _{0<x<1}\left|\frac{1}{\sum_{n=1}^{N} \frac{1}{n}} \sum_{n=1}^{N} \frac{1}{n} \chi\left(x ; x_{n}\right)-x\right|
$$

denotes the logarithmic discrepancy of $\omega$ (cf. [5]). In a recent article E. Hlawka [2] investigated the sequence $(\alpha n+\beta \log n)(\beta \neq 0)$ with respect to the logarithmic mean. He proved upper bounds for exponential sums from which (by the inequality of Erdös-Turan) we may conclude $D_{N}(\alpha n+\beta \log n) \leqslant c(\beta) / \sqrt{\log N}$. In [7] the first author proved a theorem that gives upper bounds for the discrepancy with respect to general weights and remarked that these estimates in fact give $D_{N}(\alpha n+\beta \log n) \leqslant c(\beta) / \log N$. Unfortunately the hypotheses of the theorem are not satisfied and it remains an open problem to prove this estimate (which essentially would be best possible; cf. [5] ). In the next section we prove $D_{N}(\alpha n+\beta \log n)$ $\leqslant c(\alpha, \beta) / \log N$ provided $\alpha$ is rational or of finite approximation type. In $\S 3$ we obtain $D_{N}(\alpha n+\beta \log n) \leqslant c_{1}(\beta)(\log \log N)^{2} / \log N$ by means of estimates for the exponential sums

$$
\sum_{n=1}^{N} \frac{1}{n} \mathrm{e}^{2 \pi i h(\alpha n+\beta \log n)} \quad(h=1,2, \cdots),
$$

which are accomplished by a generalization of van der Corput's method.

Notations. As usual [ $t$ ] denotes the largest integer $\leqslant t$ and the fractional part is given by $\{t\}$ $=t-[t]$; furthermore we put $\psi(t)=\{t\}-\frac{1}{2}$ and $\|t\|=\min (\{t\}, 1-\{t\})$. In $\S 2$ we make use of the notations $\lfloor t\rfloor=$ maximal integer $<t$ and $\lceil t\rceil=$ minimal integer $\geqslant t$. It is easy to see that $\lceil t\rceil=\lfloor t\rfloor+1$ and $\lceil t\rceil=-[-t]$.

\section{Elementary Estimates}

We try to establish an upper bound $c / \log N$ for $D_{N}(\omega)$, where $\omega=(\alpha n+\beta \log n)_{n=1}^{\infty}$. Given $\beta>0$ we will prove that we may choose an absolute constant $c=c(\beta)$ valid for a large class of $\alpha$ 's.

THEOREM 2.1. Assume that $0 \leqslant\{\alpha\} \leqslant c_{0} / \log N\left(c_{0}\right.$ a given positive constant). Then

$$
D_{N}(\omega) \leqslant 2 \cdot \frac{c_{0}+2+\beta+1 / \beta+\mathrm{e}^{1 / \beta}}{\log N}
$$

Proof. Let $f(u)=\{\alpha\} u+\beta \log u-\{\alpha\}$. Then $f^{\prime}(u)>0$ and $u f^{\prime}(u)=\{\alpha\} u+\beta$ is increasing.

Let $k$ be the largest integer such that $\left\lceil f^{-1}(k)\right\rceil \leqslant N$. Then we have for $0<x<1$ :

$$
\sum_{n=1}^{N} \frac{1}{n} \chi(x ; f(n))=\sum_{j=0}^{k-1} \sum_{\left\lceil f^{-1}(j)\right\rceil} \sum_{\leqslant n<\left\lceil f^{-1}(j+1)\right\rceil} \frac{1}{n} \chi(x ; f(n))+\sum_{\left\lceil f^{-1}(k)\right\rceil \leqslant n \leqslant N} \frac{1}{n} \chi(x ; f(n)) .
$$


The second sum can be estimated in the following way:

$$
\begin{aligned}
\sum_{\left[f^{-1}(k)\right\rceil \leqslant n \leqslant N} & \frac{1}{n} \chi(x ; f(n)) \leqslant \frac{N-f^{-1}(k)+1}{f^{-1}(k)} \leqslant \frac{N}{f^{-1}(k)} \leqslant \frac{f^{-1}(k+1)}{f^{-1}(k)} \\
= & \mathrm{e}^{\left(\log f^{-1}(k+1)-\log f^{-1}(k)\right)}=\mathrm{e}^{1 /\left(f^{-1}\left(\xi_{k}\right) f^{\prime}\left(f^{-1}\left(\xi_{k}\right)\right)\right.} \\
= & \mathrm{e}^{1 /\left(\{\alpha\} f^{-1}\left(\xi_{k}\right)+\beta\right)} \leqq \mathrm{e}^{1 / \beta},
\end{aligned}
$$

where $k \leqslant \xi_{k} \leqslant k+1$.

Since (for positive reals $A<B$ )

$$
\sum_{A \leqslant n<B} \frac{1}{n}=\log \frac{B}{A}+\frac{\theta}{A} \quad \text { (with }|\theta| \leqslant 1 \text { ), }
$$

we obtain

$$
\begin{aligned}
S_{j}=\sum_{\left\lceil f^{-1}(j)\right\rceil \leqslant n<\left\lceil f^{-1}(j+1)\right\rceil} \frac{1}{n} \chi(x ; f(n)) & =\sum_{f^{-1}(j) \leqslant n<f^{-1}(j+x)} \frac{1}{n} \\
& =\log f^{-1}(j+x)-\log f^{-1}(j)+\frac{\theta_{j}}{f^{-1}(j)} \quad\left(\left|\theta_{j}\right| \leqslant 1\right) .
\end{aligned}
$$

Hence, by the mean value theorem,

$$
\sum_{j=0}^{k-1} S_{j}=x \cdot \sum_{j=0}^{k-1} \frac{1}{f^{-1}\left(\xi_{x, j}\right) f^{\prime}\left(f^{-1}\left(\xi_{x, j}\right)\right)}+\theta\left(1+\int_{0}^{f(N)} \frac{d u}{f^{-1}(u)}\right)
$$

for some $\xi_{x, j}$ with $j \leqslant \xi_{x, j} \leqslant j+1$. Since

$$
\begin{aligned}
& \sum_{j=0}^{k-1} \frac{1}{f^{-1}\left(\xi_{x, j}\right) f^{\prime}\left(f^{-1}\left(\xi_{x, j}\right)\right)} \leqslant \sum_{j=0}^{k-1} \frac{1}{f^{-1}(j) f^{\prime}\left(f^{-1}(j)\right)} \\
& \leqslant \frac{1}{f^{\prime}(1)}+\int_{0}^{f(N)} \frac{d u}{f^{-1}(u) f^{\prime}\left(f^{-1}(u)\right)} \leqslant \frac{1}{\beta}+\int_{1}^{N} \frac{d t}{t}=\frac{1}{\beta}+\log N, \\
& \sum_{j=0}^{k-1} \frac{1}{f^{-1}\left(\xi_{x, j}\right) f^{\prime}\left(f^{-1}\left(\xi_{x, j}\right)\right)} \geqslant \sum_{j=0}^{k-1} \frac{1}{f^{-1}(j+1) f^{\prime}\left(f^{-1}(j+1)\right)} \\
& =\frac{-1}{f^{\prime}(1)}+\sum_{j=0}^{k} \frac{1}{f^{-1}(j) f^{\prime}\left(f^{-1}(j)\right)} \geqslant-\frac{1}{f^{\prime}(1)}+\int_{0}^{k+1} \frac{d u}{f^{-1}(u) f^{\prime}\left(f^{-1}(u)\right)} \\
& \geqslant-\frac{1}{f^{\prime}(1)}+\int_{0}^{f(N)} \frac{d u}{f^{-1}(u) f^{\prime}\left(f^{-1}(u)\right)} \geqslant-\frac{1}{\beta}+\log N
\end{aligned}
$$

and

$$
0<\int_{0}^{f(N)} \frac{d u}{f^{-1}(u)}=\int_{1}^{N} \frac{f^{\prime}(t) \mathrm{d} t}{t}=\{\alpha\} \int_{1}^{N} \frac{d t}{t}+\beta \int_{1}^{N} \frac{d t}{t^{2}} \leqslant\{\alpha\} \log N+\beta \leqslant c_{0}+\beta,
$$

we obtain 


$$
\left|\sum_{j=0}^{k-1} S_{j}-x \sum_{n=1}^{N} \frac{1}{n}\right| \leqslant 1+c_{0}+\beta+\frac{1}{\beta}+x\left|\sum_{n=1}^{N} \frac{1}{n}-\log N\right| \leqslant 2+c_{0}+\beta+\frac{1}{\beta} .
$$

Hence

$$
D_{N}(\omega) \leqslant 2 D_{N}(f(n)) \leqslant 2 \cdot \frac{c_{0}+2+\beta+1 / \beta+\mathrm{e}^{1 / \beta}}{\sum_{n=1}^{N} \frac{1}{n}},
$$

thus proving the theorem.

REMARK 1. The above arguments essentially reproduce the proof of Satz 3 in [7]. (We want to note that in the estimate for $D_{N}^{*}\left(P, x_{n}\right)$ the integral should be replaced by $\left.\int_{1}^{N} p^{\prime}(u) f^{\prime}(u) d u.\right)$

REMARK 2. By Theorem 2.1. we have

$$
D_{N}\left(\frac{n}{N}+\beta \log n ; n=1, \cdots, N\right) \leqslant 2 \cdot \frac{3+\beta+1 / \beta+\mathrm{e}^{1 / \beta}}{\log N},
$$

since $\log N / N \leqslant 1 / \mathrm{e}<1=c_{0}$.

In order to prove that $D_{N}(\omega) \leqslant c(\beta) / \log N$ uniformly for all $\alpha$ it would suffice to prove this for all rational $\alpha$ 's. This follows immediately from

$$
\left|D_{N}(\omega)-D_{N}\left(\omega^{\prime}\right)\right| \leqslant \varepsilon
$$

where $\omega=\left(x_{n}\right), \omega^{\prime}=\left(x_{n}^{\prime}\right)$ such that $\left|x_{n}-x_{n}^{\prime}\right| \leqslant \varepsilon$ (special case of [5], Satz 6). Unfortunately we did not succeed in establishing the existence of such a bound $c(\beta)$ and we must content ourselves with

THEOREM 2.2. Let $\alpha=p / q(0<p<q ; p, q$ integers $)$ and $\beta>0$. Then for $\omega=\left(x_{n}\right)=$ $((p / q) n+\beta \log n)$

$$
\left|\sum_{n=q}^{N} \frac{1}{n} \chi\left(x ; x_{n}\right)-x \sum_{n=q}^{N} \frac{1}{n}\right| \leqslant K(\beta)=\frac{2}{\beta}+2+3 \frac{\mathrm{e}^{2 / \beta}}{\mathrm{e}^{1 / \beta}-1} \quad(0 \leqslant x<1)
$$

and

$$
D_{N}(\omega) \leqq \frac{1+K(\beta)+\log q}{\log N} .
$$

Proof. Put $b=\mathrm{e}^{1 / \beta}>1$ and let $l, k$ be the largest integers such that $b^{l} \leqslant q, b^{k} \leqslant N$, respectively. Then

$$
\begin{aligned}
\sum_{n=q}^{N} \frac{1}{n} \chi\left(x ; x_{n}\right) & =-\sum_{b^{\prime}<n<q} \frac{1}{n} \chi\left(x ; x_{n}\right)+\sum_{j=l}^{k-1} \sum_{b^{j}<n<b^{j+1}} \frac{1}{n} \chi\left(x ; x_{n}\right)+\sum_{b^{k}<n<N} \frac{1}{n} \chi\left(x ; x_{n}\right) \\
& =-\mathrm{I}+\mathrm{II}+\mathrm{III} .
\end{aligned}
$$


We have for the first and the third term

$$
\mathrm{I} \leqslant \sum_{b^{l} \leqslant n<b^{l+1}} \frac{1}{n} \leqslant \log b+1, \quad \mathrm{III} \leqslant \sum_{b^{k} \leqslant n<b^{k+1}} \frac{1}{n} \leqslant \log b+1 .
$$

For the remaining term we proceed as follows:

$$
\begin{aligned}
& \mathrm{II}=\sum_{j=l}^{k-1} \sum_{r=0}^{q-1} \sum_{b^{j} \leqslant m q+r<b^{j+1}} \frac{1}{m q+r} \chi\left(x ; \frac{r p}{q}+\log _{b}(m q+r)\right) \\
& =\sum_{j=l}^{k-1} \sum_{\substack{\left\{\frac{r p}{q}\right\}<x \\
0 \leqq r<q}}\left(\sum_{\left.b^{j} \leqslant m q+r<b^{j-}-\frac{(p p}{q}\right)+x} \frac{1}{m q+r}+\sum_{\left.b^{j+1-}-\frac{r p}{q}\right\} \leqslant m q+r<b^{j+1}} \frac{1}{m q+r}\right) \\
& +\sum_{j=l}^{k-1} \sum_{\substack{\left\{\frac{r p}{q}\right\} \geq x \\
0 \leq r<q}} \sum_{b^{j+1}-\left\{\frac{p p}{q} \mid \leq m q+r<b^{j+1+x-\left\{\frac{p q}{q}\right\}}\right.} \frac{1}{m q+r} \\
& =\sum_{j=l}^{k-1} \sum_{\substack{\left\{\frac{p}{q}\right\}<x \\
0 \leq r<q}}\left(\frac{1}{q} \log b^{x}+\frac{\theta_{j r}+\theta_{j r}^{\prime}}{b^{j}}\right)+\sum_{j=l}^{k-1} \sum_{\substack{\left\{\frac{p}{q}\right\} \leq x \\
0 \leq r<q}}\left(\frac{1}{q} \log b^{x}+\frac{\theta_{j r}^{\prime \prime}}{b^{j}}\right) \\
& =(k-l) x \log b+\frac{3 \theta^{\prime} q}{1-\frac{1}{b}} \frac{1}{b^{l}}=x(\log N-\log q)+\theta^{\prime \prime} \log b+\frac{3 \theta^{\prime \prime} b^{2}}{b-1},
\end{aligned}
$$

where all $\theta$ 's are non specified numbers. with $|\theta| \leqslant 1$. Combining the estimates for I, II and III we obtain

$$
\begin{aligned}
\sum_{n=q}^{N} \frac{1}{n} \chi\left(x ; x_{n}\right) & =x(\log N-\log q)+\theta^{\prime}\left(2 \log b+1+\frac{3 b^{2}}{b-1}\right) \\
& =x \sum_{n=q}^{N} \frac{1}{n}+\theta\left(2 \log b+2+\frac{3 b^{2}}{b-1}\right) \quad(|\theta| \leqslant 1) .
\end{aligned}
$$

To prove the second part of the theorem we note that

$$
\begin{aligned}
\left|\sum_{n=1}^{N} \frac{1}{n} \chi\left(x ; x_{n}\right)-x \sum_{n=1}^{N} \frac{1}{n}\right| & \leqslant\left|\sum_{n=1}^{q-1} \frac{1}{n} \chi\left(x ; x_{n}\right)-x \sum_{n=1}^{q-1} \frac{1}{n}\right|+\left|\sum_{n=q}^{N} \frac{1}{n} \chi\left(x ; x_{n}\right)-x \sum_{n=q}^{N} \frac{1}{n}\right| \\
& \leqslant 1+\log q+2 \log b+2+\frac{3 b^{2}}{b-1}
\end{aligned}
$$

(for $N \geqslant q$, whereas the result is trivial for $N<q$ ).

REMARK 1. Taking $c_{0}=\{\alpha\} \log q$ in the last line of the proof of Theorem 2.1. we have

$$
\sum_{n=1}^{q} \frac{1}{n} D_{q}(\omega) \leqslant 2\left(\{\alpha\} \log q+2+\beta+\frac{1}{\beta}+\mathrm{e}^{1 / \beta}\right) .
$$


Hence, instead of taking the trivial bound $1+\log q$, we may write

$$
\left|\sum_{n=1}^{q-1} \frac{1}{n} \chi\left(x ; x_{n}\right)-x \sum_{n=1}^{q-1} \frac{1}{n}\right| \leqslant \frac{1}{q}+\sum_{n=1}^{q} \frac{1}{n} D_{q}(\omega) \leqslant 1+2\left(\{\alpha\} \log q+2+\beta+\frac{1}{\beta}+\mathrm{e}^{1 / \beta}\right) .
$$

This gives $D_{N}(\omega) \leqslant 2\left(\{\alpha\} \log q+\left(3+\beta+1 / \beta+K(\beta)+\mathrm{e}^{1 / \beta}\right)\right) / \log N$. As a special case we obtain a uniform bound for all $\alpha=p / q$ with $0<p / q \leqslant 1 / \log q$.

REMARK 2. Since

$$
\begin{aligned}
\left|\sum_{n=1}^{N} \frac{1}{n} \chi\left(x ; x_{n}\right)-x \sum_{n=1}^{N} \frac{1}{n}\right|= & \left|\sum_{n=1}^{N} \frac{1}{n}\left(\chi\left(x ; x_{n}\right)-x\right)\right| \leqslant\left|\frac{1}{N} \sum_{n=1}^{N}\left(\chi\left(x ; x_{n}\right)-x\right)\right| \\
& +\sum_{n=1}^{N-1} \frac{1}{n+1}\left|\frac{1}{n} \sum_{j=1}^{n} \chi\left(x ; x_{j}\right)-x\right| \leqslant \sum_{n=1}^{N-1} \frac{1}{n+1} D_{n}^{*}(\omega)+1,
\end{aligned}
$$

we have

$$
D_{N}(\omega) \leqslant \frac{1}{\log N}\left(\sum_{n=1}^{N-1} \frac{1}{n+1} D_{n}^{*}(\omega)+1\right)
$$

where $D_{n}^{*}$ denotes the usual discrepancy with respect to the arithmetic mean. In the following let $\alpha$ be an irrational number of finite approximation type $\eta \geqslant 1$, i.e. for all $\varepsilon>0$ there is a constant $c(\alpha, \varepsilon)$ such that

$$
\|q \alpha\| \geqslant \frac{c(\alpha, \varepsilon)}{q^{\eta+\varepsilon}}
$$

for all integers $q \geqslant 1$. For such $\alpha$ 's rather good estimates of $D_{N}^{*}(\alpha n)$ are known. In order to utilize these we relate $D_{N}^{*}(\omega)$ to $D_{N}^{*}(\alpha n)$. For every positive integer $h$ we have

$$
\begin{aligned}
\left|\sum_{n=1}^{N} \mathrm{e}^{2 \pi i h(\alpha n+\beta \log n)}\right|= & \left|\sum_{n=1}^{N} \mathrm{e}^{2 \pi i h \alpha n} \cdot \mathrm{e}^{2 \pi i h \beta \log n}\right| \leqslant\left|\sum_{n=1}^{N} \mathrm{e}^{2 \pi i h \alpha n}\right| \\
& +\sum_{n=1}^{N-1} 2 \pi h \beta(\log (n+1)-\log n)\left|\sum_{j=1}^{n} \mathrm{e}^{2 \pi i h \alpha j}\right| \\
\leqslant & \frac{1}{2\|h \alpha\|}(1+2 \pi \beta h \log N)
\end{aligned}
$$

since $\left|\sum_{j=1}^{n} \mathrm{e}^{2 \pi i h \alpha j}\right| \leqslant \frac{1}{|\sin \pi h \alpha|} \leqslant \frac{1}{2\|h \alpha\|}$.

Now we use the inequality of Erdös and Turan (cf. [3], p. 112) together with the estimates

$$
\sum_{h=1}^{m} \frac{1}{\|h \alpha\|} \leqslant c^{\prime}(\alpha, \varepsilon) m^{\eta+\varepsilon}, \quad \sum_{h=1}^{m} \frac{1}{h\|h \alpha\|} \leqslant c^{\prime \prime}(\alpha, \varepsilon) \cdot m^{\eta-1+\varepsilon}
$$

(cf. [3], p. 123) to obtain 


$$
\begin{aligned}
D_{N}^{*}(\omega) & \leqslant c_{0}\left(\frac{1}{m}+\sum_{h=1}^{m} \frac{1}{h}\left|\frac{1}{N} \sum_{n=1}^{N} \mathrm{e}^{2 \pi i h(\alpha n+\beta \log n)}\right|\right) \\
& \leqslant c_{0}\left(\frac{1}{m}+\frac{1}{N} \sum_{h=1}^{m} \frac{1}{2 h\|h \alpha\|}+\frac{\log N}{N} \sum_{h=1}^{m} \frac{\pi \beta}{\|h \alpha\|}\right) \\
& \leqslant c_{1}(\alpha, \varepsilon, \beta)\left(\frac{1}{m}+\frac{m^{\eta-1+\varepsilon}}{N}+\frac{\log N}{N} m^{\eta+\varepsilon}\right)
\end{aligned}
$$

Choosing $m=\left[N^{\frac{1}{n+1}}\right]$ we obtain for every $\varepsilon>0: D_{N}^{*}(\omega) \leqslant c_{2}(\alpha, \varepsilon, \beta) N^{-\frac{1}{n+1}+\varepsilon}$. Inserting this in (1) yields

$$
D_{N}(\omega) \leqslant \frac{c_{3}(\alpha, \beta)}{\log N}
$$

provided that $\alpha$ is of finite approximation type.

The argument used in the proof of Theorem 2.1. may be refined to give the following result:

THEOREM 2.3. Assume $f$ to be a twice continuously differentiable (real-valued) function defined on $[1, \infty)$ such that

$$
f(1)=0,0<c_{0} \leqslant f^{\prime}(u) \leqslant c_{1}, \quad\left|f^{\prime \prime}(u)\right| \leqslant \frac{c_{2}}{u}(u \geqslant 1) .
$$

Then for $0 \leqslant x<1$

$$
\sum_{n=1}^{N} \frac{1}{n} \chi(x ; f(n))=x \sum_{n=1}^{N} \frac{1}{n}+\sum_{j=0}^{\left[f^{-1}(N)\right]} \frac{\left\{-f^{-1}(j+x)\right\}-\left\{-f^{-1}(j)\right\}}{f^{-1}(j)}+O(1),
$$

where the $O$-constant only depends on $c_{0}, c_{1}, c_{2}$.

PROOF. Let $k$ be the largest integer such that $\left\lceil f^{-1}(k)\right\rceil \leqslant N$. Then

$$
\sum_{n=1}^{N} \frac{1}{n} \chi(x ; f(n))=\sum_{j=0}^{k-1} \sum_{\left\lceil f^{-1}(j)\right\rceil \leqslant n<\left\lceil f^{-1}(j+1)\right\rceil} \frac{1}{n} \chi(x ; f(n))+\sum_{\left\lceil f^{-1}(k)\right\rceil \leqslant n \leqslant N} \frac{1}{n} \chi(x ; f(n)) .
$$

The second sum can be estimated in the following way:

$$
\begin{aligned}
\sum_{\left\lceil f^{-1}(k)\right\rceil \leqslant n \leqslant N} \frac{1}{n} \chi(x ; f(n)) & \leqslant \frac{N-f^{-1}(k)+1}{f^{-1}(k)}<\frac{f^{-1}(k+1)-f^{-1}(k)+1}{f^{-1}(k)} \\
& \leqslant \frac{1}{f^{-1}(k) f^{\prime}\left(f^{-1}\left(\xi_{k}\right)\right.}+\frac{1}{f^{-1}(k)} \leqslant \frac{1}{c_{0}}+1 .
\end{aligned}
$$

Applying the formula

$$
\sum_{l=1}^{m} \frac{1}{l}=\gamma+\log m+\frac{1}{2 m}+O\left(\frac{1}{m^{2}}\right)
$$


we obtain

$$
\begin{aligned}
\sum_{\left\lceil f^{-1}(j)\right\rceil<n<\left\lceil f^{-1}(j+1)\right\rceil} \frac{1}{n} \chi(x ; f(n))= & \sum_{\left\lceil f^{-1}(j)\right\rceil \leqslant n \leqslant\left\lfloor f^{-1}(j+x)\right\rfloor} \frac{1}{n} \\
= & \frac{1}{\left\lceil f^{-1}(j)\right\rceil}+\log \left\lfloor f^{-1}(j+x)\right\rfloor-\log \left\lceil f^{-1}(j)\right\rceil \\
& +\frac{1}{2\left\lfloor f^{-1}(j+x)\right\rfloor}-\frac{1}{2\left\lceil f^{-1}(j)\right\rceil}+O\left(\frac{1}{\left\lceil f^{-1}(j)\right\rceil^{2}}\right)
\end{aligned}
$$

where the $O$-constants are absolute noes. We note, that the above formula is valid even if $\left\lfloor f^{-1}(j+x)\right\rfloor<\left\lceil f^{-1}(j)\right\rceil$. Next we observe that

$$
\sum_{j=0}^{\infty} \frac{1}{\left\lfloor f^{-1}(j)\right\rfloor^{2}}=O(1)
$$

Hence

$$
\begin{aligned}
\sum_{n=1}^{N} \frac{1}{n} \chi(x ; f(n))= & \sum_{j=0}^{k-1}\left(\log f^{-1}(j+x)-\log f^{-1}(j)\right. \\
& \left.+\frac{\left\lceil f^{-1}(j+x)\right\rceil-f^{-1}(j+x)}{f^{-1}(j)}-\frac{\left\lceil f^{-1}(j)\right\rceil-f^{-1}(j)}{f^{-1}(j)}\right)+O(1),
\end{aligned}
$$

using Taylor's theorem, formula (3) and $\lceil t\rceil=\lfloor t\rfloor+1$. The $O$-constants depend on $c_{0}, c_{1}$, $c_{2}$. Since $\lceil t\rceil-t=-[-t]-t=\{-t\}$, the result may be written as

$$
\sum_{j=0}^{k-1}\left(\log f^{-1}(j+x)-\log f^{-1}(j)\right)+\sum_{j=0}^{[f-1(N)]} \frac{\left\{-f^{-1}(j+x)\right\}-\left\{-f^{-1}(j)\right\}}{f^{-1}(j)}+O(1) .
$$

By Taylor's theorem,

$$
\begin{aligned}
\log f^{-1}(j+x) & -\log f^{-1}(j) \\
& =x \frac{1}{f^{-1}(j) f^{\prime}\left(f^{-1}(j)\right)}+\frac{x^{2}}{2}\left(-\frac{f^{-1}(\xi) f^{\prime \prime}\left(f^{-1}(\xi)\right)+f^{\prime}\left(f^{-1}(\xi)\right)}{f^{-1}(\xi)^{2} f^{\prime}\left(f^{-1}(\xi)\right)^{3}}\right)
\end{aligned}
$$

(for some $\xi$ with $j \leqslant \xi \leqslant j+x$ ). The absolute value of the last expression is bounded above by

$$
\frac{f^{-1}(\xi)\left|f^{\prime \prime}\left(f^{-1}(\xi)\right)\right|+c_{1}}{f^{-1}(\xi)^{2} c_{0}^{3}} \leqslant \frac{c_{2}+c_{1}}{f^{-1}(j)^{2} c_{0}^{3}}=O\left(\frac{1}{f^{-1}(j)^{2}}\right) .
$$

Hence (applying (3)) we obtain by Euler's summation formula

$$
\sum_{j=0}^{k-1}\left(\log f^{-1}(j+x)-\log f^{-1}(j)\right)=x \int_{1}^{k-1} \frac{d t}{f^{-1}(t) f^{\prime}\left(f^{-1}(t)\right)}+O(1) .
$$

The proof of the theorem is now completed by observing that 


$$
\int_{1}^{k-1} \frac{d t}{f^{-1}(t) f^{\prime}\left(f^{-1}(t)\right)}=\log f^{-1}(k-1)-\log f^{-1}(1)=\sum_{n=1}^{N} \frac{1}{n}+O(1) .
$$

REMARK. The above theorem may be applied to the sequence $\omega=(\alpha n+\beta \log n)(\alpha$, $\beta \geqslant 0)$; we just have to consider $f(u)=(1+\{\alpha\}) u+\beta \log u-(1+\{\alpha\})$. The existence of a bound $D_{N}(\omega) \leqslant c^{\prime}(\beta) / \log N$ (uniformly in $\alpha$ ) is equivalent to

$$
\left|\sum_{j=0}^{M} \frac{\left\{-f^{-1}(j+x)\right\}-\left\{-f^{-1}(j)\right\}}{f^{-1}(j)}\right| \leqslant c(\beta) \text { for all } M, \alpha \text { and } x .
$$

\section{Exponential Sums}

In the following we give a refinement of [2], Satz 1 in the case of the logarithmic mean.

THEOREM 3.1. For reals $\alpha, \beta(\beta \neq 0)$ and positive integers $h$ we have

$$
\left|\sum_{n=A}^{B} \frac{1}{n} \mathrm{e}^{2 \pi i h(\alpha n+\beta \log n)}\right| \leqslant C(\beta)\left(\frac{\sqrt{h}}{A}+\frac{1}{\sqrt{h}}\right),
$$

and

$$
\left|\sum_{n=1}^{N} \frac{1}{n} \mathrm{e}^{2 \pi i h(\alpha n+\beta \log n)}\right| \leqslant \log h+1+2 C(\beta),
$$

where $A, B$ and $N$ denote positive integers and $C(\beta)$ is a constant depending continuously on $\beta$.

PROOF. We begin by showing how to deduce the second formula form the first.

$$
\begin{aligned}
\left|\sum_{n=1}^{N} \frac{1}{n} \mathrm{e}^{2 \pi i h(\alpha n+\beta \log n)}\right| & \leqslant \sum_{n=1}^{h} \frac{1}{n}+\left|\sum_{n=h+1}^{N} \frac{1}{n} \mathrm{e}^{2 \pi i h(\alpha n+\beta \log n)}\right| \\
& \leqslant 1+\log h+C(\beta)\left(\frac{\sqrt{h}}{h+1}+\frac{1}{\sqrt{h}}\right) \leqslant 1+\log h+2 C(\beta) .
\end{aligned}
$$

For the main part of the proof we require the following lemma:

LEMMA 1. Let $A<B$ be positive reals. Then for arbitrary reals $\alpha, \beta(\beta \neq 0)$ we have

$$
\begin{aligned}
& \left|\int_{A}^{B} \frac{1}{u} \mathrm{e}^{2 \pi i h(\alpha u+\beta \log u)} d u\right| \leqslant\left(1+\frac{1}{\sqrt{\pi|\beta|}}\right) \frac{16}{\sqrt{\pi h|\mathrm{~b}|}}, \\
& \left|\int_{A}^{B} \frac{1}{u^{2}} \mathrm{e}^{2 \pi i h(\alpha u+\beta \log u)} d u\right| \leqslant\left(1+\frac{\sqrt{2}}{\sqrt{\pi|\beta|}}\right) \frac{32}{A \sqrt{\pi h|\beta|}}
\end{aligned}
$$

for all positive integers $h$.

Proof of the Lemma. We may restrict ourselves to the case $\beta>0$; otherwise we may 
take the complex conjugate of the integral. If $\alpha u+\beta \neq 0$ for all $u$ with $A \leq u \leq B$ then

$$
\left|\int_{A}^{B} \frac{1}{u} \mathrm{e}^{2 \pi i h(\alpha u+\beta \log u)} d u\right|=\left|\int_{A}^{B} \frac{\left(\mathrm{e}^{2 \pi i h(\alpha u+\beta \log u)^{\prime}}\right.}{2 \pi i h(\alpha u+\beta)} d u\right| .
$$

By the second mean value theorem we obtain

$$
\begin{aligned}
\left|\int_{A}^{B} \frac{\left(\mathrm{e}^{2 \pi i h(\alpha u+\beta \log u)^{\prime}}\right.}{\alpha u+\beta} d u\right| \leqslant\left|\int_{A}^{B} \frac{(\cos 2 \pi h(\alpha u+\beta \log u))^{\prime}}{\alpha u+\beta} d u\right| \\
+\left|\int_{A}^{B} \frac{(\sin 2 \pi h(\alpha u+\beta \log u))^{\prime} d u}{\alpha u+\beta}\right| \leqslant 2^{3} \max _{A<u<B} \frac{1}{|\alpha u+\beta|} .
\end{aligned}
$$

Assume that $u_{0}=-\beta / \alpha \leqslant A$. Then for $0<\varepsilon<1$ we have

$$
\begin{aligned}
\left|\int_{A}^{B} \frac{1}{u} \mathrm{e}^{2 \pi i(\alpha u+\beta \log u)} d u\right| & \leqslant \int_{A}^{(1+\varepsilon) A} \frac{d u}{u}+\left.\right|_{(1+\varepsilon) A} ^{(1+\varepsilon) B} \frac{1}{u} \mathrm{e}^{2 \pi i h(\alpha u+\beta \log u)} d u \mid+\int_{B}^{(1+\varepsilon) B} \frac{d u}{u} \\
& \leqslant 2 \log (1+\varepsilon)+\frac{4}{\pi h \varepsilon \beta},
\end{aligned}
$$

since

$$
\max _{(1+\varepsilon) A<u<1+\varepsilon) B} \frac{1}{|\alpha u+\beta|} \leqslant \frac{1}{\left|\alpha(1+\varepsilon) u_{0}+\beta\right|}=\frac{1}{\varepsilon \beta} .
$$

Let us now assume that $B \leqslant u_{0}$. Then for $0<\varepsilon<1$ we have

$$
\begin{aligned}
\left|\int_{A}^{B} \frac{1}{u} \mathrm{e}^{2 \pi i h(\alpha u+\beta \log u)} d u\right| & \leqslant \int_{(1-\varepsilon) A}^{A} \frac{d u}{u}+\left.\right|_{(1-\varepsilon) A} ^{(1-\varepsilon) B} \frac{1}{u} \mathrm{e}^{2 \pi i h(\alpha u+\beta \log u)} d u \mid+\int_{(1+\varepsilon) B}^{B} \frac{d u}{u} \\
& \leqslant 2 \log \frac{1}{1-\varepsilon}+\frac{4}{\pi h \varepsilon \beta},
\end{aligned}
$$

since

$$
\max _{(1-\varepsilon) A<<<(1-\varepsilon) B} \frac{1}{|\alpha u+\beta|} \leqslant \frac{1}{\left|\alpha(1+\varepsilon) u_{0}+\beta\right|}=\frac{1}{\varepsilon \beta} .
$$

Hence for arbitrary $A, B, \varepsilon$ with $0<A<B, 0<\varepsilon<1$ we obtain

$$
\left|\int_{A}^{B} \frac{1}{u} \mathrm{e}^{2 \pi i h(\alpha u+\beta \log u)} d u\right| \leqslant 2 \log \frac{1+\varepsilon}{1-\varepsilon}+\frac{8}{\pi h \varepsilon \beta} .
$$

Choosing $\varepsilon=1 / \sqrt{\pi h \beta}$ for $h \geqslant 4 / \pi \beta$ we obtain the upper bound $8(\varepsilon+1 / \pi h \varepsilon \beta)=16 / \sqrt{\pi h \beta}$, since

$$
\log \frac{1+\varepsilon}{1-\varepsilon}=\log \left(1+\frac{2}{1-\varepsilon}\right) \leqslant \frac{2 \varepsilon}{1-\varepsilon} \leqslant 4 \varepsilon \text { for } 0<\varepsilon \leqslant \frac{1}{2} .
$$

For $1 \leqslant h<4 / \pi \beta$ we choose $\varepsilon=1 / 2$ and obtain the bound $8(1 / 2+2 / \pi h \beta)$ 
$<8(2 / \sqrt{\pi h \beta}+2 / \pi \sqrt{h} \beta)$. Thus the first part of the Lemma is proved.

For the second integral let us start again with the case $\alpha u+\beta \neq 0$ for all $u$ with $A \leqslant u \leqslant B$. Then, as above, we obtain

$$
\left|\int_{A}^{B} \frac{1}{u^{2}} \mathrm{e}^{2 \pi i h(\alpha u+\beta \log u)} d u\right| \leqslant \frac{1}{2 \pi h} \cdot 2^{4} \max _{A \leqslant u \leqslant B} \frac{1}{|u(\alpha u+\beta)|},
$$

since $1 / u(\alpha u+\beta)$ consists of at most two monotone pieces.

If $u_{0}=-\beta / \alpha \leqslant A$, then for $0<\varepsilon<1$ we have

$$
\begin{aligned}
\left|\int_{A}^{B} \frac{1}{u^{2}} \mathrm{e}^{2 \pi i h(\alpha u+\beta \log u)} d u\right| & \leqslant \int_{A}^{(1+\varepsilon) A} \frac{d u}{u^{2}}+\left|\int_{(1+\varepsilon) A}^{(1+\varepsilon) B} \frac{1}{u^{2}} \mathrm{e}^{2 \pi i h(\alpha u+\beta \log u)} d u\right|+\int_{B}^{(1+\varepsilon) B} \frac{d u}{u^{2}} \\
& \leqslant \frac{\varepsilon}{1+\varepsilon} \cdot \frac{1}{A}+\frac{8}{(1+\varepsilon) A \pi h \varepsilon \beta}+\frac{\varepsilon}{1+\varepsilon} \cdot \frac{1}{B} \leqslant \frac{2}{(1+\varepsilon) A}\left(\varepsilon+\frac{4}{\pi h \varepsilon \beta}\right),
\end{aligned}
$$

since

$$
\max _{(1+\varepsilon) A<u<(1+\varepsilon) B} \frac{1}{|u(\alpha u+\beta)|} \leqslant \frac{1}{(1+\varepsilon) A} \cdot \frac{1}{\left|\alpha(1+\varepsilon) u_{0}+\beta\right|}=\frac{1}{(1+\varepsilon) A \varepsilon \beta} .
$$

Similarly, for $B \leqslant u_{0}$ we obtain the bound $2 /(1-\varepsilon) A \cdot(\varepsilon+4 / \pi h \varepsilon \beta)$. Hence for arbitrary $A$, $B, \varepsilon$ with $0<A<B, 0<\varepsilon<1$ we may take the upper bound $4 /\left(1-\varepsilon^{2}\right) A \cdot(\varepsilon+4 / \pi h \varepsilon \beta)$. For $h \geqslant 8 / \pi \beta$ we choose $\varepsilon=2 / \sqrt{\pi h \beta} \leqslant 1 / \sqrt{2}$ and obtain the upper bound $8 / A \cdot 4 / \sqrt{\pi h \beta}$; choosing $\varepsilon=1 / \sqrt{2}$ for $h<8 / \pi \beta$ gives the bound $8 / A \cdot(1 / \sqrt{2}+4 \sqrt{2} / \pi h \beta$ ). Since (for $1 \leqslant h<8 / \pi \beta$ ) $1+\sqrt{2 / \pi \beta}>\sqrt{\pi h \beta} / 4 \sqrt{2}+\sqrt{2 / \pi h \beta}=\sqrt{\pi h \beta}(1 / \sqrt{2}+4 \sqrt{2} / \pi h \beta) / 4$, this yields the second inequality of the Lemma.

In the proof of the previous lemma we have shown the following estimates

LEMMA 2. If $\alpha u+\beta \neq 0$ for $0<A \leqslant u \leqslant B$ then

$$
\begin{aligned}
& \left|\int_{A}^{B} \frac{1}{u} \mathrm{e}^{2 \pi i h(\alpha u+\beta \log u)} d u\right| \leqslant \frac{4}{\pi h} \max _{A \leqslant u \leqslant B} \frac{1}{|\alpha u+\beta|} \\
& \left|\int_{A}^{B} \frac{1}{u^{2}} \mathrm{e}^{2 \pi i h(\alpha u+\beta \log u)} d u\right| \leqslant \frac{8}{A \pi h} \max _{A \leqslant u \leqslant B} \frac{1}{|\alpha u+\beta|} .
\end{aligned}
$$

We continue now with the proof of the theorem. By Euler's summation formula

$$
\begin{aligned}
\sum_{n=A}^{B} \frac{1}{n} \mathrm{e}^{2 \pi i h(\alpha n+\beta \log n)=} & \frac{\theta}{A}+\int_{A}^{B} \frac{1}{u} \mathrm{e}^{2 \pi i h(\alpha u+\beta \log u)} d u \\
& +\int_{A}^{B} \psi(u)\left(-\frac{1}{u^{2}}+\frac{1}{u} 2 \pi i h\left(\alpha+\frac{\beta}{u}\right)\right) \mathrm{e}^{2 \pi i h(\alpha u+\beta \log u)} d u
\end{aligned}
$$

for some complex number $\theta$ with $|\theta| \leqslant 1$. We may assume that $1 \leqslant \alpha<2$ and $\beta>0$, since for $\beta<0$ we may take the complex conjugate of the exponential sum and the sum remains un- 
changed if we replace $\alpha \beta \xi \alpha+k$ for integral $k$. By Lemma 2

$$
\left|\int_{A}^{B} \frac{1}{u} \mathrm{e}^{2 \pi i h(\alpha u+\beta \log u)} d u\right| \leqslant \frac{4}{\pi h(\alpha+\beta)}
$$

The trivial estimate $|\psi(u)| \leqslant 1 / 2$ yields

$$
\left|\int_{A}^{B} \frac{\psi(u)}{u^{2}} \mathrm{e}^{2 \pi i h(\alpha u+\beta \log u)} d u\right| \leqslant \frac{1}{2 A} .
$$

Using the Fourier expansion of $\psi(u)$ we obtain

$$
\begin{aligned}
\int_{A}^{B} \psi(u) & \frac{2 \pi i h}{u}\left(\alpha+\frac{\beta}{u}\right) \mathrm{e}^{2 \pi i h(\alpha u+\beta \log u)} d u \\
= & \int_{A}^{B} \sum_{m \neq 0} \frac{2 \pi i h}{m}\left(\frac{\alpha}{u}+\frac{\beta}{u^{2}}\right) \mathrm{e}^{2 \pi i m u} \mathrm{e}^{2 \pi i h(\alpha u+\beta \log u)} d u \\
= & \sum_{m \neq 0} \frac{2 \pi i h}{m} I_{m}\left(I_{m}=\int_{A}^{B}\left(\frac{\alpha}{u}+\frac{\beta}{u^{2}}\right) \mathrm{e}^{2 \pi i h\left(\left(\alpha+\frac{m}{h}\right) u+\beta \log u\right)} d u\right) .
\end{aligned}
$$

Put

$$
\begin{aligned}
& M_{1}=\left\{m \in \mathbb{Z}: m<-\left(1+h\left(\alpha+\frac{\beta}{A}\right)\right) \text { or } m>-h \alpha+1, m \neq 0\right\}, \\
& M_{2}=\left\{m \in \mathbb{Z}:-\left(1+h\left(\alpha+\frac{\beta}{A}\right)\right) \leqslant m \leqslant-h \alpha+1\right\} .
\end{aligned}
$$

For

$$
m<-\left(1+h\left(\alpha+\frac{\beta}{A}\right)\right) \text { we have } \alpha+\frac{m}{h}<-\frac{1}{h}-\frac{\beta}{A}<0
$$

and $(\alpha+m / h) A+\beta<-A / h<0$. Hence for these $m$

$$
\max _{A \leqslant u<B} \frac{1}{\left|\left(\alpha+\frac{m}{h}\right) u+\beta\right|}=\frac{1}{\left|\left(\alpha+\frac{m}{h}\right) A+\beta\right|} .
$$

For $m>-h \alpha+1(m \neq 0)$ we have $\alpha+m / h>0$ and

$$
\max _{A<u<B} \frac{1}{\left|\left(\alpha+\frac{m}{h}\right) u+\beta\right|}=\frac{1}{\left(\alpha+\frac{m}{h}\right) A+\beta} .
$$

Thus Lemma 2 gives

$$
\left|I_{m}\right| \leqslant\left(\frac{4 \alpha}{\pi h}+\frac{8 \beta}{A \pi h}\right) \frac{1}{\left|\left(\alpha+\frac{m}{h}\right) A+\beta\right|} \leqslant \frac{4(\alpha+2 \beta)}{A \pi\left|m+h\left(\alpha+\frac{\beta}{A}\right)\right|} \text { for } m \in M_{1} \text {. }
$$

For $m \in M_{2}$ we obtain 


$$
\left|I_{m}\right| \leqslant \frac{16}{\sqrt{\pi h \beta}}\left(1+\frac{\sqrt{2}}{\sqrt{\pi \beta}}\right)(\alpha+2 \beta)
$$

by Lemma 1 . As card $\left(M_{2}\right) \leqslant 3+h \beta / A$, we derive

$$
\begin{aligned}
& \left|\sum_{m \neq 0} \frac{2 \pi i h}{m} I_{m}\right| \leqslant 2 \pi h\left(\sum_{m \in M_{1}} \frac{4(\alpha+2 \beta)}{\pi A\left|m+h\left(\alpha+\frac{\beta}{A}\right)\right||m|}+\frac{3+h \beta / A}{h \alpha / 2} \frac{16}{\sqrt{\pi h \beta}}\right. \\
& \left.\times\left(1+\frac{\sqrt{2}}{\sqrt{\pi \beta}}\right)(\alpha+2 \beta)\right) \\
& =\frac{8(\alpha+2 \beta)}{A}\left(h \sum_{m \in M_{1}} \frac{1}{|m|\left|m+h\left(\alpha+\frac{\beta}{A}\right)\right|}+\frac{8(3+h \beta / A) A \pi}{\alpha \sqrt{h \beta \pi}}\left(1+\frac{2}{\pi \beta}\right)\right) .
\end{aligned}
$$

Put $\mu=h(\alpha+\beta / A)$, then

$$
\begin{aligned}
\sum_{m \in M_{1}} \frac{1}{|m||m+\mu|} & =\sum_{m>\mu+1} \frac{1}{m(m-\mu)}+\sum_{0<m<h \alpha-1} \frac{1}{m(\mu-m)}+\sum_{m>0} \frac{1}{m(m+\mu)} \\
& \leqslant 2 \sum_{m=1}^{\infty} \frac{1}{m(m+\mu)}+\sum_{0<m<h \alpha-1} \frac{1}{m(\mu-m)} .
\end{aligned}
$$

We have $\mu>1$ (since $h, \alpha \geq 1$ ), and so

$$
\sum_{m=1}^{\infty} \frac{1}{m(m+\mu)} \leqslant \sum_{m=1}^{\infty} \frac{1}{m(m+[\mu])}=\frac{1}{[\mu]} \sum_{m=1}^{[\mu]} \frac{1}{m} \leqslant 2 \frac{1+\log \mu}{\mu} .
$$

Since

$$
\sum_{0<m<h \alpha-1} \frac{1}{m(\mu-m)}=\frac{1}{\mu} \sum_{0<m<h \alpha-1}\left(\frac{1}{\mu-m}+\frac{1}{m}\right) \leqslant \frac{2}{\mu} \sum_{0<m<[\mu]} \frac{1}{m} \leqslant 2 \frac{1+\log \mu}{\mu},
$$

we obtain

$$
\sum_{m \in M_{1}} \frac{1}{|m||m+\mu|} \leqslant 6 \frac{1+\log \mu}{\mu} \leqslant 6 \frac{1+\log (h(\alpha+\beta))}{h \alpha}
$$

Hence

$$
\left|\sum_{m \neq 0} \frac{2 \pi i h}{m} I_{m}\right| \leqslant \frac{8(\alpha+2 \beta)}{A}\left(6 h \frac{1+\log (h(\alpha+\beta)}{h \alpha}+\frac{8(3+h \beta / A) A \pi}{\alpha \sqrt{h \beta \pi}}\left(1+\frac{\sqrt{2}}{\sqrt{\pi \beta}}\right)\right),
$$

and so

$$
\begin{aligned}
& \left|\sum_{n=A}^{B} \frac{1}{n} \mathrm{e}^{2 \pi i h(\alpha n+\beta \log n)}\right| \leqslant \frac{1}{A}+\frac{4}{\pi h(\alpha+\beta)}+\frac{1}{2 A} \\
& \quad+\frac{8(\alpha+2 \beta)}{A}\left(6 h \frac{1+\log (h(\alpha+\beta))}{h \alpha}+\frac{8(3+h \beta / A) A \pi}{\alpha \sqrt{h \beta \pi}}\left(1+\frac{\sqrt{2}}{\sqrt{\pi \beta}}\right)\right) \leqslant C(\beta) \cdot\left(\frac{\sqrt{h}}{A}+\frac{1}{\sqrt{h}}\right),
\end{aligned}
$$

where $C(\beta)$ is a constant only depending on $\beta$. Thus the proof of the theorem is complete. 
COROLLARY. For reals $\alpha, \beta(\beta \neq 0)$ we have

$$
D_{N}(\alpha n+\beta \log n) \leqslant C_{1}(\beta) \frac{(\log \log N)^{2}}{\log N} .
$$

PROOF. We choose $m=[\log N]+1$ in the inequality of Erdös-Turan for the logarithmic mean (cf. [4], Th. 1):

$$
D_{N}\left(x_{n}\right) \leqq 4\left(\frac{1}{m}+\sum_{h=1}^{m} \frac{1}{h}\left|\frac{1}{\sum_{n=1}^{N} \frac{1}{n}} \sum_{n=1}^{N} \frac{1}{n} \mathrm{e}^{2 \pi i h x_{n}}\right|\right) .
$$

A simple calculation yields the desired result.

Exponential sums for functions of a similar type as $\alpha n+\beta \log n$ can be related to the exponential sums considered above by the following theorem.

THEOREM 3.2. Let $p(n)$ be positive weights and let $\left(x_{n}\right),\left(a_{n}\right)$ be sequences of real numbers and assume that $\left|a_{n+1}-a_{n}\right| \leqslant c / n^{1+\delta} P(n)$ (with positive constants $c$ and $\delta$ ), where $P(n)=$ $\sum_{k=1}^{n} p(k)$. Then we have for positive values $h$

$$
\left.\mid \sum_{n=1}^{N} p(n) \mathrm{e}^{2 \pi i h\left(x_{n}+a_{n}\right.}\right)|\leqslant 2 P(m)+| \sum_{n=1}^{N} p(n) \mathrm{e}^{2 \pi i h x_{n}}\left|+2 \pi c \sum_{n=m+1}^{N} \frac{1}{n^{1+\delta / 2}}\right| \frac{1}{P(n)} \sum_{n=1}^{n} p(k) \mathrm{e}^{2 \pi i h x_{k}} \mid,
$$

where $m=\left[h^{2 / \delta}\right]+1$.

PROOF. Since $\left|\mathrm{e}^{i u}-\mathrm{e}^{i v}\right| \leqslant|u-v|$ we have

$$
\begin{aligned}
& \left|\sum_{n=1}^{N} p(n) \mathrm{e}^{2 \pi i h\left(x_{n}+a_{n}\right)}\right| \leqslant \sum_{n=1}^{m} p(n)+\mid \sum_{n=m+1}^{N}\left(\sum_{k=1}^{n} p(k) \mathrm{e}^{2 \pi i h x_{k}}\right)\left(\mathrm{e}^{2 \pi i h a_{n}}-\mathrm{e}^{2 \pi i h a_{n+1}}\right) \\
& \quad-\sum_{k=1}^{m} p(k) \mathrm{e}^{2 \pi i h x_{k}} \mathrm{e}^{2 \pi i h a_{m+1}}+\sum_{k=1}^{N} p(k) \mathrm{e}^{2 \pi i h x_{k}} \mathrm{e}^{2 \pi i h a_{N+1}} \mid \\
& \leqslant 2 \sum_{n=1}^{m} p(n)+\sum_{n=m+1}^{N}\left|\sum_{k=1}^{n} p(k) \mathrm{e}^{2 \pi i n x_{k}}\right| 2 \pi h\left|a_{n}-a_{n+1}\right|+\left|\sum_{n=1}^{N} p(n) \mathrm{e}^{2 \pi i h x_{n}}\right| \\
& \leqslant 2 \sum_{n=1}^{m} p(n)+\sum_{n=m+1}^{N}\left|\sum_{k=1}^{n} p(k) \mathrm{e}^{2 \pi i h x_{k}}\right| \frac{2 \pi c \cdot n^{\delta / 2}}{n^{1+\delta} P(n)}+\left|\sum_{n=1}^{N} p(n) \mathrm{e}^{2 \pi i h x_{n}}\right|,
\end{aligned}
$$

thus proving the theorem.

Assuming $\left|a_{n+1}-a_{n}\right| \leqslant c p(n) / P(n)$ instead of $\left|a_{n+1}-a_{n}\right| \leqslant c / n^{1+\delta} P(n)$ we obtain (for $m=0$ )

$$
\left|\frac{1}{P(N)} \sum_{n=1}^{N} p(n) \mathrm{e}^{2 \pi i h\left(x_{n}+a_{n}\right)}\right| \leqslant \frac{2 \pi h c}{P(N)} \sum_{n=1}^{N} p(n)\left|\frac{1}{P(n)} \sum_{k=1}^{n} p(k) \mathrm{e}^{2 \pi i h x_{k}}\right|+\left|\frac{1}{P(N)} \sum_{n=1}^{N} p(n) \mathrm{e}^{2 \pi i h x_{n}}\right| .
$$


Hence $\lim _{N \rightarrow \infty} P(N)^{-1} \sum_{n=1}^{N} p(n) \mathrm{e}^{2 \pi i h x_{n}}=0$ implies $\lim _{N \rightarrow \infty} P(N)^{-1} \sum_{n=1}^{N} p(n) \mathrm{e}^{2 \pi i h\left(x_{n}+a_{n}\right)}=0$ provided that $\lim _{N=\infty} P(N)=\infty$. Thus Weyl's criterion (cf. [1], p. 55) gives

THEOREM 3.3. Let $p(n)$ be positive weights with $\lim _{N \rightarrow \infty} \sum_{n=1}^{N} p(n)=\infty$. If $\left(x_{n}\right)$ is uniformly distributed with respect to the weights $p(n)$ and if $\left|a_{n+1}-a_{n}\right|=O\left(p(n) / \sum_{k=1}^{n} p(k)\right.$ then $\left(x_{n}+a_{n}\right)$ is also uniformly distributed with respect to $p(n)$.

COROLLARY. For $\omega=\left(\alpha n+\beta \log n+a_{n}\right)$ with reals $\alpha, \beta(\beta \neq 0)$ and $\left|a_{n+1}-a_{n}\right| \leqslant c / n^{1+\delta}$ $(c, \delta$ positive constants) we have

$$
D_{N}(\omega) \leqslant C(\beta, c, \delta) \frac{(\log \log N)^{2}}{\log N}
$$

where $D_{N}$ denotes the discrepancy with respect to the logarithmic mean. $(C(\beta, c, \delta)$ may be chosen to depend continuously on $\beta$.)

PROOF. From Theorem 3.1. and Theorem 3.2. we easily deduce (for $x_{n}=\alpha n+\beta \log n$ )

$$
\left|\sum_{n=1}^{N} \frac{1}{n} \mathrm{e}^{2 \pi i h\left(x_{n}+a_{n}\right)}\right| \leqslant c_{1}(\beta, c, \delta)(\log h+1) .
$$

As in the proof of the Corollary after Theorem 3.1., the assertion follows from the inequality of Erdös and Turan.

REMARK 1. In the special case of the arithmetic mean $p(n)=1$ the result of Theorem 3.3. can be found in [6].

REMARK 2. From Theorem 2.3. and the corollary after Theorem 3.1. it follows that (for $N \geqslant 2$ )

$$
\sum_{j=0}^{\left[f^{-1}(N)\right]} \frac{\left\{-f^{-1}(j+x)\right\}-\left\{-f^{-1}(j)\right\}}{f^{-1}(j)} \leqslant C(\beta)(\log \log N)^{2},
$$

where $f^{-1}$ denotes the inverse function of $\alpha x+\beta \log x-\alpha(1 \leqslant \alpha<2, \beta>0)$ and $C(\beta)$ is a constant only depending on $\beta$. In the following we give an upper bound for $D_{N}\left(\omega^{*}\right)$, where $\omega^{*}=\left(f^{-1}(n)\right)_{n=1}^{\infty}$. We define $\varepsilon(y)$ by

$$
x=\frac{y}{\alpha}-\frac{\beta}{\alpha} \log \frac{y}{\alpha}+\varepsilon(y), \quad \alpha x+\beta \log x=y+\alpha .
$$

Since $d y / d x=\alpha+\beta / x$, we obtain

$$
\varepsilon^{\prime}(y)=\frac{x}{\alpha x+\beta}-\frac{1}{\alpha}+\frac{\beta}{\alpha} \frac{1}{y}=\frac{\beta}{\alpha} \frac{\alpha x+\beta-y}{y(\alpha x+\beta)}=\frac{\beta}{\alpha} \frac{\beta(1-\log x)+\alpha}{y(\alpha x+\beta)} ;
$$


thus

$$
\begin{aligned}
\left|\varepsilon^{\prime}(y)\right| & \leqslant \frac{\beta}{\alpha} \frac{\beta \log x+\beta+\alpha}{\alpha x \cdot y} \leqslant\left(\frac{\beta}{\alpha}\right)^{2} \frac{\log (y / \alpha+1)+1+\alpha / \beta}{(y+\alpha) y}(\alpha+\beta) \\
& \leqslant \beta^{2}(1+\beta)\left(1+\frac{1}{\beta}\right) \frac{\log (y+1)+1}{y^{2}}
\end{aligned}
$$

(where we have used the trivial estimates $\alpha x \leqslant y+\alpha<(\alpha+\beta) x$ ). By the mean value theorem we have

$$
|\varepsilon(n+1)-\varepsilon(n)| \leqslant \beta(1+\beta)^{2} \frac{\log (n+2)+1}{n^{2}} \leqslant \frac{3 \beta(1+\beta)^{2}}{n^{3 / 2}} .
$$

Applying the last corollary to the sequence $\omega^{*}=(n / \alpha-\beta / \alpha \cdot \log n+\beta / \alpha \cdot \log \alpha+\varepsilon(n))$ yields

$$
D_{N}\left(\omega^{*}\right) \leqslant \max _{1<\alpha<2} C\left(-\frac{\beta}{\alpha}, 3 \beta(1+\beta)^{2}, \frac{1}{2}\right) \frac{(\log \log N)^{2}}{\log N}=c_{0}(\beta) \frac{(\log \log N)^{2}}{\log N} .
$$

\section{References}

[1] Hlawka, E., Theorie der Gleichverteilung, Bibl. Inst., Mannheim-Wien-Zürich, 1979.

[2] Hlawka, E., Gleichverteilung und Konvergenzverhalten von Potenzreihen am Rande des Konvergenzkreises, Manuscripta Math. 44 (1983) 231-263.

[3] Kuipers, L. and Niederreiter, H., Uniform Distribution of Sequences, John Wiley and Sons, New York, 1974.

[4] Niederreiter, H. and Philipp, W., Berry-Esseen-bounds and a theorem of Erdös and Turan on uniform distribution mod 1, Duke Math. J. 40 (1973) 633-649.

[5] Niederreiter, H. and Tichy, R. F., Beiträge zur Diskrepanz bezüglich gewichteter Mittel, Manuscripta Math. 42 (1983) 85-99.

[6] Rindler, H., Fast konstante Folgen, Acta Arithm. 35 (1979) 189-193.

[7] Tichy, R. F., Diskrepanz bezüglich gewichteter Mittel und Konvergenzverhalten von Potenzreihen, Manuscripta Math. 44 (1983) 265-277.

[8] Tsuji, M., On the Uniform Distribution of Numbers mod. 1, J. Math. Soc. Japan 4 (1952) 313322.

[9] Weyl, H., Über die Gleichverteilung von Zahlen mod. Eins, Math. Ann. 77 (1916) 313-352.

Institut für Analysis, Technische Mathematik und Versicherungsmathematik.

Technical University Vienna.

Wiedner Hauptstraße 6-10, A-1040 Vienna, Austria.

Current address of G. Turnwald:

Mathematisches lnstitut der Universität,

Auf der Morgenstelle 10,

D-7400 Tübingen

Federal Republic of Germany 